\title{
Quality of life after laparoscopic sentinel node navigation surgery in early gastric cancer: a single-center cohort study
}

\author{
Sang II Youn ${ }^{1} \cdot$ Sang Yong Son ${ }^{2} \cdot$ Kanghaeng Lee ${ }^{1} \cdot$ Yongjoon Won ${ }^{1} \cdot$ Sahong Min ${ }^{1} \cdot$ Young Suk Park ${ }^{1}$. \\ Sang-Hoon Ahn ${ }^{1} \cdot$ Hyung-Ho Kim ${ }^{1}$ (D)
}

Received: 12 August 2020 / Accepted: 18 November 2020 / Published online: 3 January 2021

(C) The International Gastric Cancer Association and The Japanese Gastric Cancer Association 2021

\begin{abstract}
Objective To investigate the quality of life (QOL) of patients after laparoscopic sentinel node navigation surgery (SNNS) compared to conventional laparoscopy-assisted distal gastrectomy (LADG) in early gastric cancer patients.

Methods Patients recruited for laparoscopic SNNS surgery between July 2010 and April 2013 were assessed for their QOL. A historical control group was established, consisting of patients who underwent conventional LADG with radical lymphadenectomy from the same institution. QOL questionnaire was taken serially from preoperative week 1 until 12 months postoperatively $(1,3,6$, and 12 months) using the Korean version of the European Organization for Research and Treatment of Cancer (EORTC) QOL questionnaire-core (QLQ-C30) and gastric cancer-specific questionnaire (STO22).

Results A total of 80 prospectively gathered patients who received SNNS were categorized into the comparison group (SNNS group). The QOL was compared with 78 patients identified to have received LADG from the gastric cancer database of our institution and were sorted into the control group (LADG group). In QLQ-C30, SNNS group showed better functioning scales in all except role functioning and better scores from the symptom scales in fatigue, insomnia, and diarrhea compared to the LADG group. In QLQ-STO22, scores on dysphagia, eating restriction, anxiety, and body image disturbance were better in SNNS group.

Conclusions Postoperative QOL in laparoscopic gastrectomy combined with SNNS is superior compared to conventional laparoscopic distal gastrectomy in patients with stage I gastric cancer.
\end{abstract}

Keywords Gastric cancer · Laparoscopic gastrectomy $\cdot$ Sentinel lymph node $\cdot$ Quality of life

\section{Introduction}

Current standard treatment for gastric cancer is gastrectomy with radical lymphadenectomy [1-3]. It is needless to mention that ensuring the oncologic safety through sufficient excision is critical to effective treatment. However, with recent outcomes of clinical trials proving that there is no benefit from excessively radical surgery $[4,5]$, the current paradigm of treatment for early gastric cancer has shifted from performing radical surgery, to attaining both oncologic

Hyung-Ho Kim

hhkim@snubh.org

1 Department of Surgery, Seoul National University Bundang Hospital, Gumi-ro 173-82, Bundang-gu, Seongnam, Gyenggi-do 463-707, Republic of Korea

2 Department of Surgery, Ajou University Hospital, Suwon-Si, Korea safety and postoperative quality of life (QOL) of patients by means of minimally invasive surgery.

Laparoscopic surgery is a procedure created in the backgrounds of minimally invasive surgery. Advantages of laparoscopic gastrectomy include better short term outcomes and postoperative QOL compared to open gastrectomy [6]. Nowadays, laparoscopy in gastric surgery is popularized and has advanced to a point where guidelines recommend laparoscopic surgery over laparotomy in EGC [2].

Sentinel lymph node navigation surgery (SNNS) is also a procedure that was created in efforts to reduce the invasiveness and minimize the extent of surgery. Main part of this procedure includes injecting a dye around the tumor, which can help accurately visualize and remove the sentinel lymph nodes [7]. The initial concept of sentinel lymph node biopsy which started from the treatment of malignant melanoma and breast cancer $[8,9]$ has extended to be utilized in gastric cancer. 
Earlier attempts of SNNS was carried out by using indocyanine green (ICG) as a sole tracer. Dual tracer method is a technique introduced by Kitagawa et al. using both dye and radioisotope, which contributed to a step forward towards accurate detection of lymph nodes [10]. It has been proven to be a reliable method for predicting lymph node metastasis in small gastric adenocarcinomas with mucosal or submucosal invasion. A study result from our institution using the Dual method also demonstrated technical feasibility and oncological safety in cT1N0M0 gastric cancer patients [11].

Aside from the oncologic outcomes, the effect of SNNS on the quality of life of patients undergoing surgery for early gastric cancers is currently unknown. By reducing the extent of lymphadenectomy and potentially, the extent of gastrectomy by utilizing SNNS, we anticipated improvement in the postoperative QOL during the follow-up period. This study, therefore, is aimed to investigate the difference in QOL of patients who received SNNS compared to conventional LADG.

\section{Methods}

\section{Patients and study design}

We assessed the QOL of patients who underwent laparoscopic SNNS surgery between July 2010 and April 2013. Patients who underwent limited gastrectomy after showing negative findings in the sentinel nodes and have answered the questionnaires properly during this period were assorted into the "SNNS group". To evaluate the QOL of patients in the SNNS group, we established a historical-comparative control group consisting of patients who underwent conventional LADG with radical lymphadenectomy. We collected the information of the patients who received conventional LADG from the database of Seoul National University Bundang Hospital (SNUBH) from June 2006 to September 2008, and categorized them into the "LADG group". The baseline selection criteria for the constituents of LADG group was patients who received LADG in an identical manner with SNNS group except for the SNNS procedure.

The common inclusion criteria were as follows: (1) age, 20 to 80 years; (2) clinical stage, T1N0M0 and pathological stage T1-2N0M0 according to the AJCC/UICC 7th edition. The QOL of the SNNS group and LADG group was longitudinally compared.

All participants were fully informed and written informed consents were obtained for the study. The institutional review board of Seoul National University Bundang Hospital approved this study (No. B-0812/065-007) and was conducted in accordance with the Declaration of Helsinki.

\section{Surgical procedures of SNNS}

The surgical procedure of SNNS was introduced from our previous study [12]. To introduce the procedure briefly, simultaneous indocyanine green (ICG) and technetium-99 m-antimony sulfur colloid (99mTc ASC)-guided laparoscopic SNNS was performed. A total volume of $4 \mathrm{~mL}$ of mixed dual tracers $(4.0 \mathrm{~mL}$ of $5 \mathrm{~g} / \mathrm{L}$ ICG and $1 \mathrm{mCi}$ of $99 \mathrm{mTc}$ ASC) was injected evenly in four quadrants of the submucosal layer of the tumor via intraoperative endoscopy. Within 15 min after endoscopic injection, the operator detected stained lymph nodes (green nodes) and radioactive lymph nodes (hot nodes), which have radioactivity equivalent to $>10$ times that of the background activity. Hot and/or green nodes were defined as the sentinel lymph nodes (SNs). Then, sentinel basins (SBs), which are lymphatic compartments containing SNs, were dissected and extracted. Bench work was carried out at a separate back table to pick up the SNs and the non-SNs, and all dissected SNs were transferred to a pathologist for frozen sectioning and permanent pathologic examination. If no SNs were identified during bench work, non-SNs were used for frozen sectioning.

If all SNs were negative in the frozen sectioning (SNnegative group), laparoendoscopic limited resection of the stomach was performed for removal of the primary lesion. Intraoperative endoscopic submucosal dissection (ESD), laparoscopic wedge resection, segmental resection (not including the pylorus in the resection range), and hemigastrectomy (including the pylorus) were performed depending on the location and size of the primary lesion. If any metastasis was detected in SNs (SN-positive group), the operation was converted to laparoscopic distal gastrectomy with radical lymphadenectomy.

\section{Assessment of QOL}

The patient QOL was evaluated using the Korean version of the European Organization for Research and Treatment of Cancer (EORTC) QOL questionnaire-core (QLQ-C30) and gastric cancer-specific questionnaire (STO22). The questionnaire was taken prospectively for both SNNS and LADG groups; once preoperatively and then serially on postoperative $1,3,6$, and 12 months. When more than $30 \%$ of the data from the questionnaire was missing the patient was excluded from the study, and if less than $30 \%$, the missing scores were substituted by the median value for that specific question [13]. Of the $89 \mathrm{SN}$ negative patients, 80 patients $(89.8 \%)$ answered to the questionnaires properly and were enrolled in the QOL analysis. We identified 78 patients who satisfy our inclusion criteria from the 
gastric cancer database of Seoul National University Bundang Hospital and included them in the present analysis.

From the EORTC-QLQ-C30, five functional scales (physical, role, cognitive, emotional, and social), a global health status, three symptom scales (fatigue, pain, and nausea/vomiting), five single items for common symptoms reported by cancer patients (dyspnea, appetite loss, insomnia, constipation, and diarrhea), were obtained. From the EORTC QLQSTO22 one functional scale (body image), five symptom scales (dysphagia, eating restriction, pain, reflux symptoms, and anxiety), and two single symptom items (dry mouth, taste) were recorded.

All scores are on a scale of 1 to 100 . A higher score for the functioning scale represents better functioning, and a higher score for the symptom scale denotes an increased symptom severity.

The QOL evaluation using DAUGS-32 or PGSAS-45 would have been a correct selection of the questionnaire since they were developed specifically for post-gastrectomy patients. However, the only validated questionnaire available in those days was EORTC-QLQ-C30 and STO22 which was the best alternative that we were able to use.

\section{Statistical analysis}

Analysis of clinicopathological characteristics was done through Student t-test for continuous variables and chisquare test for categorical variables. The QOL scores taken at each follow-up was compared to the baseline preoperative score, and the difference was calculated for every follow-up period. Statistical analysis with respect to the overall changes of the QOL between the two groups was done through a generalized estimating equation using the unstructured covariance matrix. SPSS software (version 18.0; SPSS Inc, Chicago, Ill) and R-statistics, version 3.1.1 (R Foundation for Statistical Computing, Vienna, Austria) was used for all analyses. A $p$-value of $\leq 0.05$ was considered statistically significant.

\section{Results}

\section{Baseline characteristics}

From July 2010 and April 2013, a total of 80 patients were recruited into the SNNS group. Among the 80 patients, 8 patients underwent ESD via intraoperative gastroscopy, 8 received wedge resection, 53 received segmental resection, and 11 received hemigastrectomy. The SNNS group was compared with the LADG group, consisting of 78 patients selected from the SNUBH database (Fig. 1).

The general characteristics and the differences between the two groups are summarized in Table 1. Regarding the tumor

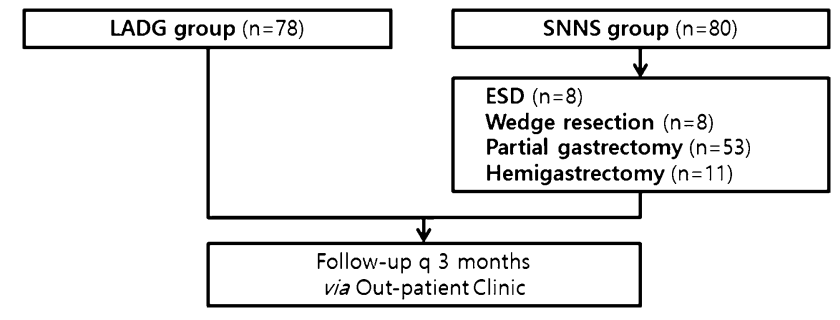

Fig. 1 Flow chart of patient selection and analysis

features, the mean tumor size of LADG group was larger than that of SNNS group $(p=0.030)$ but there was no difference in the histological type $(p=0.322)$ and tumor depth $(p=0.111)$. There was no difference in postoperative complications in both groups with 3 cases each. Time to soft diet was significantly faster in the SNNS group $(p<0.001)$ while time to flatus showed no difference $(p=0.152)$.

\section{Quality of life after laparoscopic SNNS}

The comparison of EORTC QLQ-C30 and QLQ-STO22 scores during the 12 months of follow-up is summarized in Table 2. The changes in QOL scores at each time point is displayed as a diagram with overall significance in Fig. 2a, b. In the QLQ-C30, global health status $(p<0.001)$, physical functioning $(p=0.010)$, emotional functioning $(p<0.001)$, cognitive functioning $(p=0.024)$, fatigue $(p<0.001)$, insomnia $(p=0.006)$, and diarrhea $(p=0.007)$ were statistically better in the SNNS group. In QLQ-STO22, dysphagia $(p=0.009)$, eating restriction $(p=0.006)$, anxiety $(p<0.001)$, and body image disturbance $(p=0.003)$ was statistically better in the SNNS group.

In subgroup analysis, we divided the SNNS group into two groups according to the extent of gastric resection: ESD with wedge resection $(n=16)$ and segmental resection with hemigastrectomy $(n=64)$. ESD and wedge resection group showed better QLQ-C30 scores than segmental resection and hemigastrectomy group in global health status $(p<0.001)$, cognitive functioning $(p=0.012)$, social functioning $(p=0.012)$, nausea and vomiting ( $p=0.024)$, dyspnea $(p=0.008)$, and insomnia $(p=0.018)$. For QLQ-STO22, scores were statistically better in SNNS regarding pain $(p=0.039)$, reflux symptoms $(p=0.001)$, eating restriction $(p=0.045)$, dry mouth $(p=0.021)$, and taste $(p<0.001)$. The diagram of the change in QOL for the subgroup is shown in Fig. 3a, b.

\section{Discussion}

The greatest advantage of SNNS is the benefit from function preservation through limited gastrectomy. These benefits include lowering the incidence of dumping syndrome 
Table 1 Clinicopathological characteristics and surgical outcomes: SNNS vs. LADG

\begin{tabular}{llll}
\hline Characteristics & SNNS $(n=80),(\%)$ & LADG $(n=78),(\%)$ & $p$-value \\
\hline Age (mean \pm SD $*)($ years) & $59.7 \pm 12.1$ & $56.9 \pm 10.5$ & 0.118 \\
Gender ratio (M:F) & $51: 29$ & $56: 22$ & 0.280 \\
Comorbidity & $36 / 80(45.0)$ & $26 / 78(33.3)$ & 0.133 \\
Operative procedure & $8 / 8 / 53 / 11$ & & $<0.001$ \\
ESD/WR/SR/Hemigastrectomy & & $2.40 \pm 1.29$ & 0.030 \\
Tumor size (cm) & $1.96 \pm 1.22$ & & 0.111 \\
Tumor depth & & $49(62.8 \%)$ & \\
Mucosal & $61(76.3 \%)$ & $23(29.4 \%)$ & \\
Submucosal & $17(21.3 \%)$ & $6(7.8 \%)$ & $<0.001$ \\
Proper muscle & $2(2.5 \%)$ & $39.0 \pm 12.3$ & 0.322 \\
Retrieved LNs & $16.4 \pm 11.9$ & $48(61.5 \%)$ & \\
Histology & $43(53.8 \%)$ & $30(38.5 \%)$ & 1.000 \\
Differentiated & $37(46.2 \%)$ & & 0.401 \\
Undifferentiated & $3(3.8 \%)$ & $3(3.8 \%)$ & $<0.001$ \\
Postoperative complication & $198.7 \pm 38.1$ & $205.1 \pm 60.6$ & 0.152 \\
Operative time (min) & $2.8 \pm 0.7$ & $3.3 \pm 0.6$ & $3.2 \pm 0.9$ \\
Soft diet (day) & $3.0 \pm 0.7$ & & \\
Flatus passage (day) & & & \\
\hline
\end{tabular}

SNNS: sentinel node navigation surgery, LADG: laparoscopy assisted distal gastrectomy, ESD: Endoscopic submucosal resection, WR: Wedge resection, SR: Segmental resection, RY: Roux-en-Y

Table 2 Quality of Life between SNNS and LADG

\begin{tabular}{|c|c|c|c|c|c|c|c|c|c|c|c|}
\hline & \multirow[t]{2}{*}{$p$ value } & \multicolumn{2}{|c|}{ Baseline } & \multicolumn{2}{|c|}{ POD 1 month } & \multicolumn{2}{|c|}{ POD 3 months } & \multicolumn{2}{|c|}{ POD 6 months } & \multicolumn{2}{|c|}{ POD 12 months } \\
\hline & & SNNS & LADG & SNNS & LADG & SNNS & LADG & SNNS & $\overline{\mathrm{LADG}}$ & SNNS & LADG \\
\hline \multicolumn{12}{|l|}{ QLQ-C30 } \\
\hline Global functioning & $0.001 *$ & 62.20 & 70.71 & 59.75 & 57.90 & 65.29 & 69.28 & 67.97 & 74.37 & 69.01 & 69.61 \\
\hline Physical functioning & $0.010 *$ & 86.83 & 88.15 & 77.16 & 72.49 & 84.61 & 81.45 & 86.55 & 86.87 & 87.13 & 88.28 \\
\hline Role functioning & 0.180 & 89.50 & 89.12 & 71.27 & 63.29 & 85.52 & 80.58 & 86.82 & 87.69 & 87.23 & 86.19 \\
\hline Emotional functioning & $<0.001 *$ & 74.42 & 82.54 & 80.77 & 81.13 & 84.25 & 84.00 & 85.22 & 87.24 & 86.66 & 80.54 \\
\hline Cognitive functioning & $0.024 *$ & 88.59 & 89.88 & 90.59 & 85.63 & 87.38 & 83.54 & 86.90 & 89.41 & 86.34 & 83.72 \\
\hline Social functioning & $0.002 *$ & 82.74 & 86.79 & 81.55 & 74.67 & 86.86 & 85.47 & 84.78 & 89.81 & 89.09 & 86.58 \\
\hline Fatigue & $<0.001 *$ & 28.92 & 23.65 & 34.03 & 39.32 & 25.35 & 31.04 & 27.19 & 24.56 & 22.22 & 27.61 \\
\hline Nausea \& Vomiting & 0.362 & 7.72 & 5.38 & 19.19 & 8.60 & 8.49 & 6.04 & 7.36 & 7.44 & 6.54 & 7.92 \\
\hline Pain & 0.585 & 8.71 & 9.03 & 22.05 & 26.90 & 12.82 & 11.79 & 10.99 & 8.69 & 8.84 & 6.47 \\
\hline Dyspnea & 0.262 & 6.96 & 11.46 & 12.08 & 20.01 & 8.16 & 12.31 & 6.34 & 7.98 & 7.20 & 8.28 \\
\hline Insomnia & $0.006^{*}$ & 23.62 & 13.64 & 23.34 & 23.82 & 15.45 & 11.51 & 17.23 & 9.81 & 12.97 & 13.83 \\
\hline Appetite loss & 0.063 & 15.29 & 11.50 & 36.45 & 37.58 & 11.16 & 14.05 & 14.96 & 10.44 & 10.07 & 13.89 \\
\hline Constipation & 0.262 & 14.82 & 8.49 & 23.60 & 24.28 & 15.91 & 8.91 & 12.25 & 9.81 & 16.59 & 6.47 \\
\hline Diarrhea & $0.007 *$ & 10.88 & 11.46 & 14.26 & 17.86 & 12.05 & 20.40 & 11.82 & 18.41 & 17.10 & 25.83 \\
\hline \multicolumn{12}{|l|}{ QLQ-STO22 } \\
\hline Dysphagia & $0.009 *$ & 5.79 & 5.38 & 15.95 & 22.22 & 7.00 & 10.18 & 7.10 & 5.09 & 5.66 & 4.89 \\
\hline Pain & 0.921 & 14.86 & 13.77 & 24.39 & 26.55 & 15.75 & 15.31 & 15.25 & 13.35 & 14.16 & 12.19 \\
\hline Reflux symptoms & 0.152 & 13.34 & 10.74 & 23.51 & 17.85 & 14.75 & 11.29 & 17.11 & 12.64 & 14.36 & 11.67 \\
\hline Eating restrictions & $0.006 *$ & 8.59 & 7.57 & 23.26 & 25.95 & 12.73 & 16.00 & 10.33 & 12.04 & 8.07 & 10.64 \\
\hline Anxiety & $<0.001 *$ & 25.17 & 12.91 & 29.78 & 24.85 & 26.55 & 22.79 & 23.75 & 16.17 & 19.37 & 17.47 \\
\hline Dry mouth & 0.063 & 18.13 & 17.00 & 28.32 & 28.99 & 14.62 & 20.46 & 14.52 & 17.83 & 13.29 & 21.22 \\
\hline Taste & 0.116 & 37.03 & 38.18 & 52.40 & 57.64 & 42.69 & 48.13 & 36.73 & 41.76 & 38.81 & 39.56 \\
\hline Body image disturbance & $0.003 *$ & 15.72 & 13.63 & 28.73 & 29.37 & 21.80 & 28.99 & 18.14 & 20.91 & 15.17 & 13.83 \\
\hline
\end{tabular}

*Statistically significant $(p<0.05)$, SNNS sentinel node navigation surgery, LADG laparoscopy assisted distal gastrectomy, POD Postoperative day, Numbers in the table represent the mean score and the leftmost column represents the $p$ value drawn from Generalized Estimating Equation 

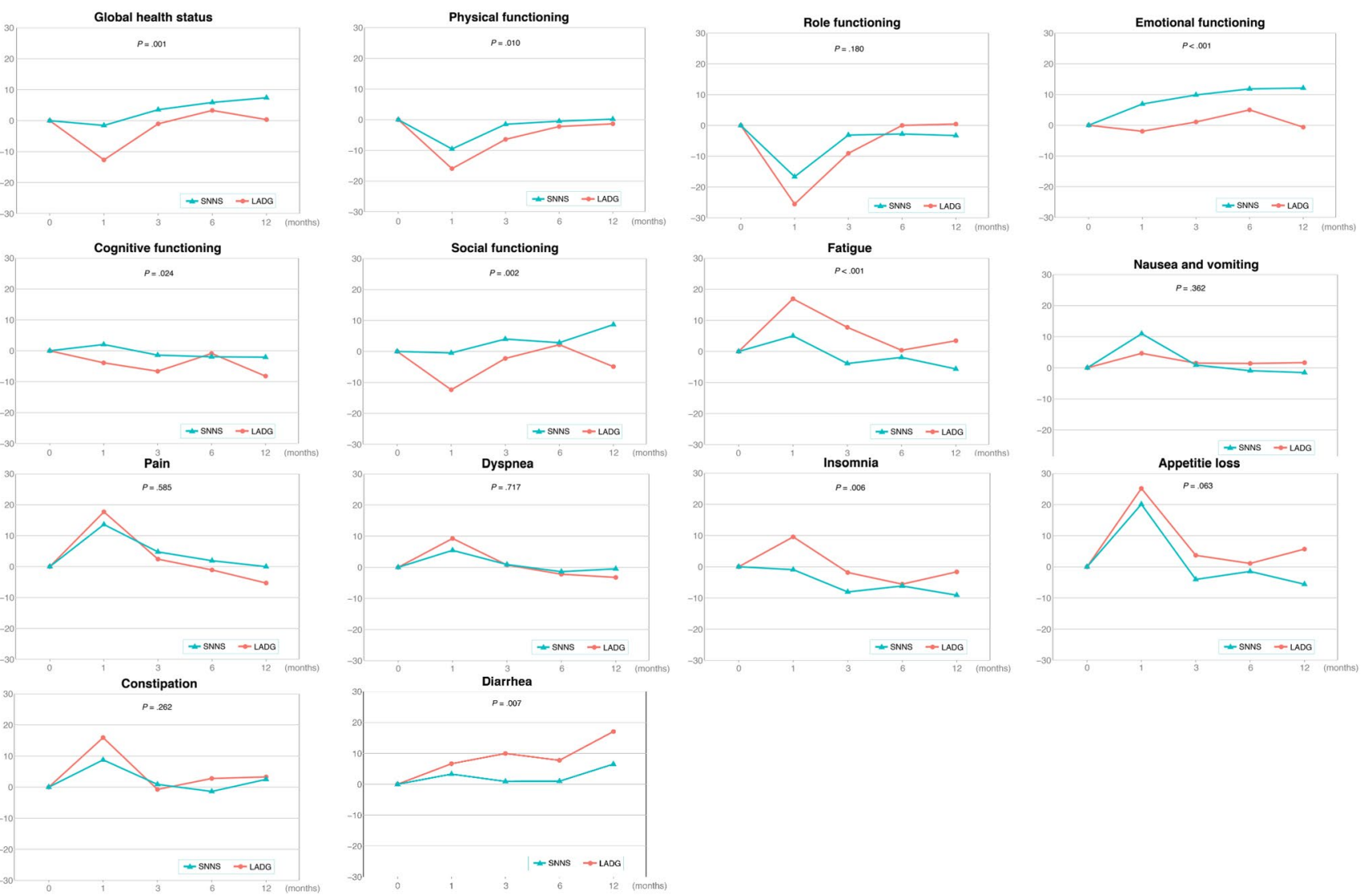

Fig. 2 Quality of life for LADG vs. SNNS
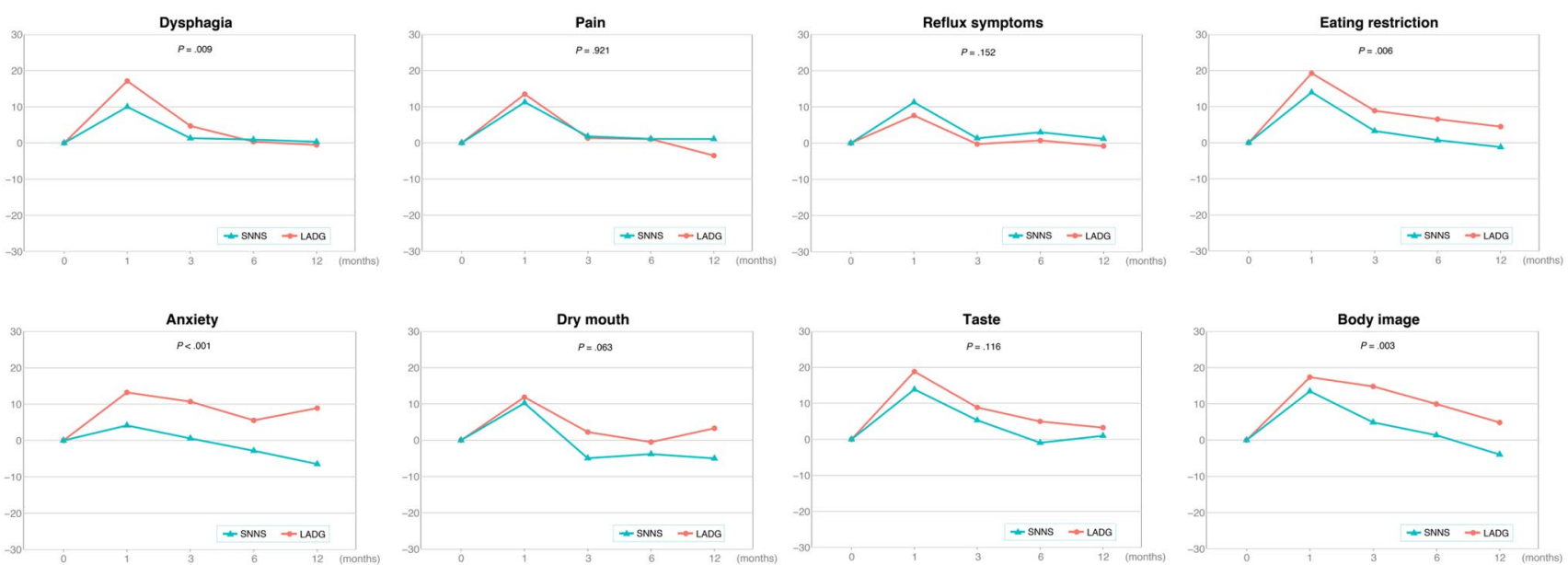

Fig. 2 (continued)

[14] and less post-gastrectomy syndromes [15] through pylorus-preserving gastrectomy. Apart from these objective results, the significance of performing limited surgery would be diminished if there is no subjective feedback from the patients. Hence, the postoperative QOL results of the patients convey great significance in SNNS.
Even though QOL is an important aspect of SNNS, oncologic safety must not be compromised. Many studies are reporting SNNS to be technically feasible and oncologically safe. Our prior phase II study on SNNS for EGC patients revealed that laparoscopic SNNS can be oncologically safe in EGC patients [12]. Yaguchi et al. also reported long term 


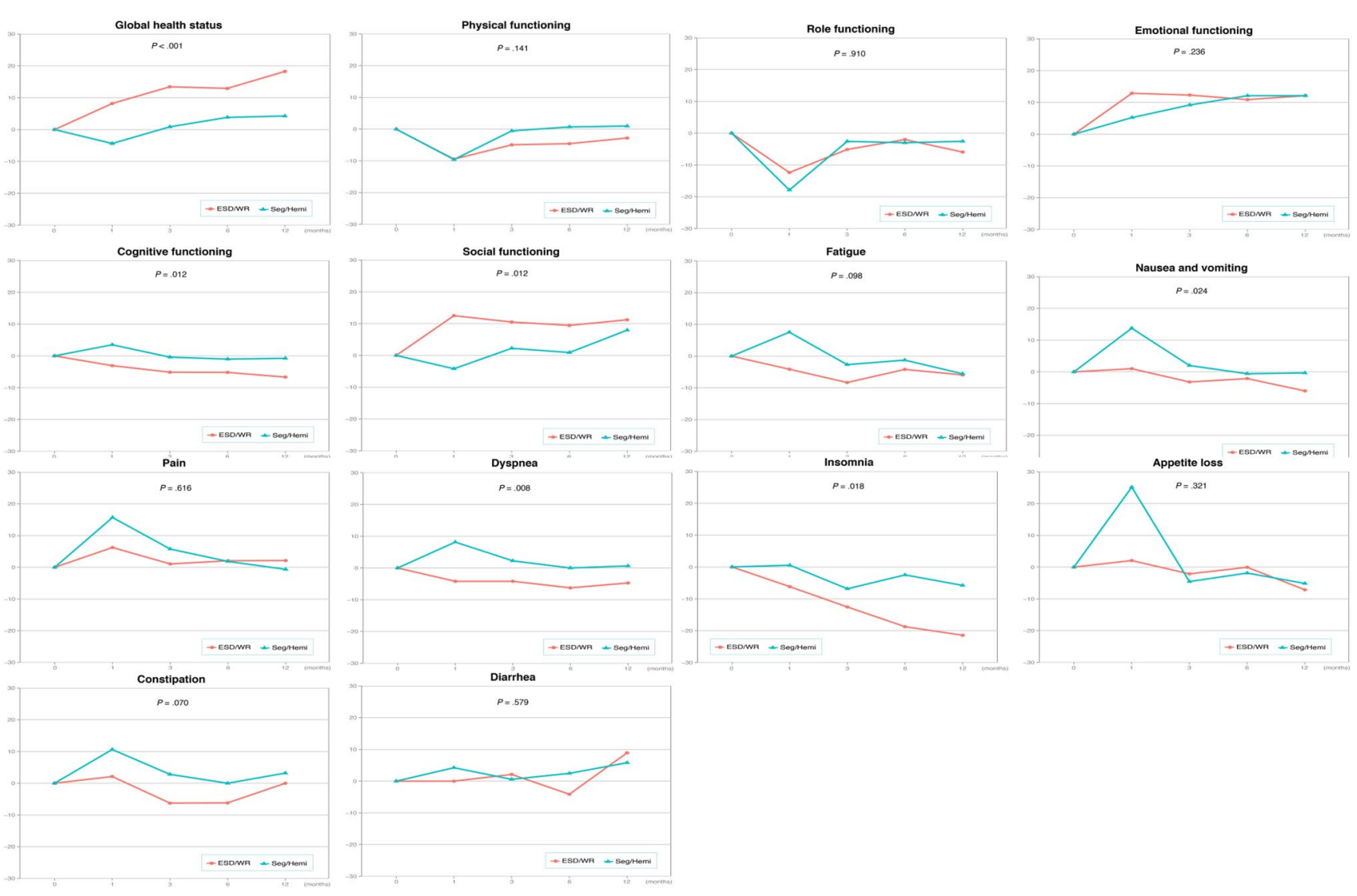

Fig. 3 Quality of life for ESD or Wedge resection vs. Segmental resection or Hemigastrectomy
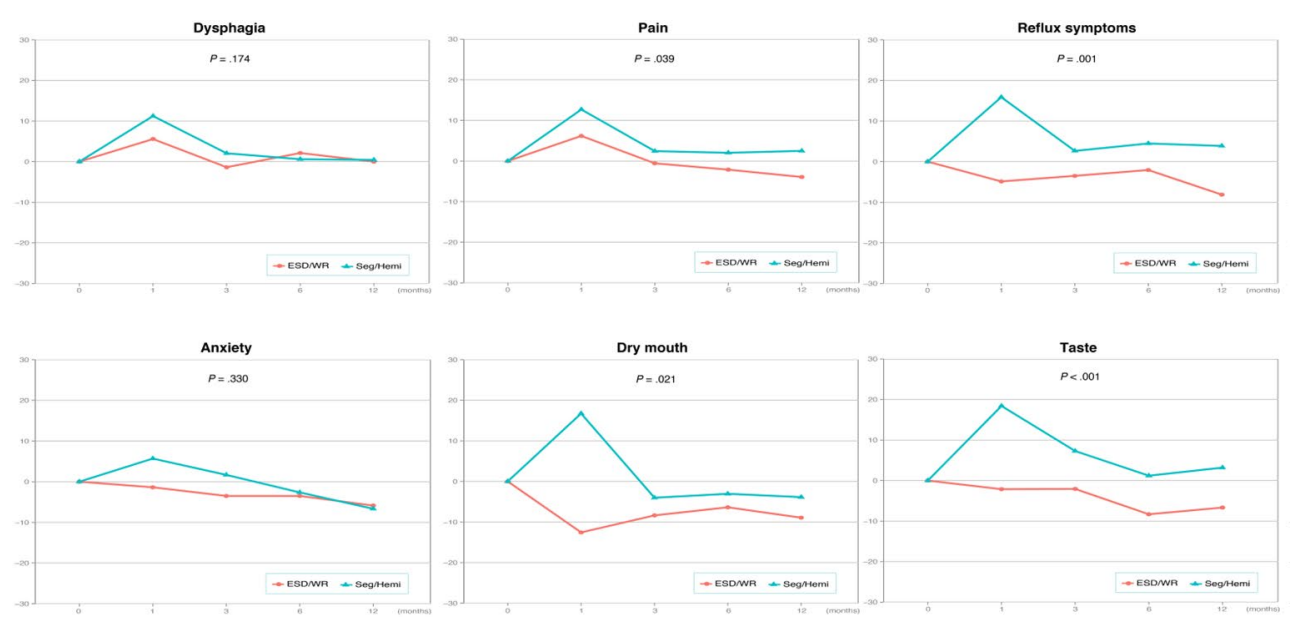
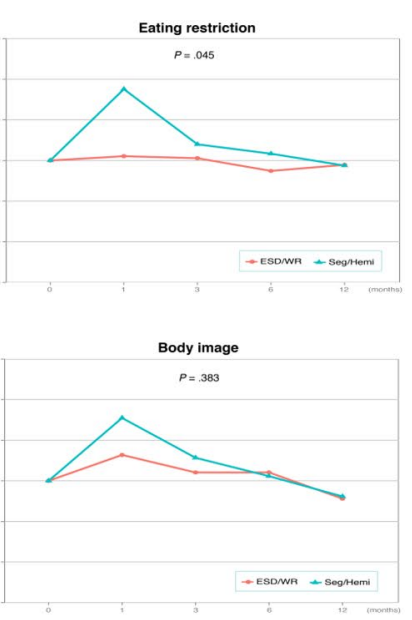

Fig. 3 (continued)

outcome for SNNS stating that it can be useful over conventional gastrectomy [16]. A multicenter study in Japan also confirmed that dual tracer method is safe and effective when applied to early-stage gastric cancer [17]. Thereby, SNNS is beginning to be applied to gastric cancer in more and more institutions.
The diagram in Fig. 2a, b shows a general tendency of the QOL index to deteriorate sharply in postoperative 1-month, irrelevant to the type of procedure the patient received, and gradually recovers after time. The QOL value is markedly superior in the SNNS group than LADG group in most categories in terms of lesser exacerbation and faster recovery, 
even when the difference was not statistically significant. The benefit of SNNS is especially prominent in that the QOL index related to oral intake, which includes eating restriction, dysphagia, and diarrhea is better in the SNNS group. The SNNS allows surgeons to inflict less surgical damage to the vagal innervation through minimal manipulation of the surrounding tissue, which presumably resulted in less post-gastrectomy symptoms.

Although the majority of the categories showed superior values in the SNNS group, there are some parameters that were not statistically significant; some of these categories include role functioning, dyspnea, nausea/vomiting, reflux symptoms, and pain. Another tendency from the QOL diagram that we can observe from these categories is that they all tended to show similar QOL scores preoperatively and postoperatively, resulting in a relatively flat shaped diagram. A flat diagram connotes that the item was not affected by the surgery, which would most likely result in no statistical difference.

Looking into the details, our present study did not reveal any difference in pain between the SNNS group and LADG group. A previous study comparing the severity of pain between LADG than open distal gastrectomy [18] reported significantly less pain from patients who received LADG. The difference in pain had much association with the amount of skin incision and wound traction during surgery. Meanwhile, LADG and SNNS share an identical procedure of making a laparoscopic incision. Therefore, the absence of difference in pain between the LADG and SNNS groups, shows that reducing the extent of lymph node dissection does not contribute to the amelioration of pain.

In addition, the categories related to nausea/vomiting, and reflux symptoms showed little difference between the two groups, which is quite unusual. Since the scores in both LADG group and SNNS group showed little difference before and after the surgery, a questionnaire more specific to post-gastrectomy QOL evaluation, such as DAUGS-32 or PGSAS-45, might have given a more accurate analysis related to post gastrectomy syndrome.

The SNNS group consists of four differently treated groups, with all four treatments not involving standard lymph node dissection. To assess the effect of reducing the scope of gastric resection on QOL, we conducted a subgroup analysis by grouping the ESD with Wedge resection $(n=16)$ and Segmental resection with Hemigastrectomy $(n=64)$. The results in Fig. 3a, b also show that the QOL of ESD/ Wedge resection group is better than Segmental resection/ Hemi gastrectomy group, denoting that limiting the amount of stomach resection clearly has a positive impact on postoperative QOL. Isozaki et al. reported the postoperative QOL after limited gastrectomy and concluded that better QOL can be achieved through limited gastrectomy [18]. The result of our subgroup analysis is along the same line, with the addition that this could be safely achieved through SNNS. A study from Wu et al. also reported that changes of QOL are largely affected by the scope of gastric resection and disease status, rather than the extent of lymph node dissection [19]. Another study comparing QOL between patients who received ESD and standard gastrectomy also concluded that ESD provides better QOL in the early post-treatment period [20].

Despite our positive results for SNNS, this study has several limitations. Firstly, even though prospectively collected data were used for SNNS patients, the data for the LADG group was historically gathered and retrospectively analyzed, creating an inevitable bias in the results. Secondly, the laparoscopic experience of the surgeon has advanced over time, which could have affected the result. To minimize the effect of improvement in surgeon proficiency, we had to compare the SNNS group to a historical group with a similar type of gastrectomy done in the simultaneous period. While LADG was the main procedure in that period, gastrectomy performed after the patients enrolled with SNNS were mainly done with a totally laparoscopic method with automated staplers, both of which could create bias.

Several studies have previously been conducted to evaluate the QOL after SNNS and demonstrated positive results $[21,22]$. Our study is also another attempt to explore the benefits of SNNS which may contribute to a better understanding of SNNS. Further prospective randomized studies in a multicenter scale are needed for more concrete evidence for the effectiveness of SNNS in gastrectomy. The SENORITA trial [23] is a large scale multicenter phase III randomized control trial with patients randomized to laparoscopic stomach-preserving surgery with SBD or standard surgery. This study is yet to report the outcomes, which is expected to give a more in-depth analysis of SNNS.

In conclusion, postoperative QOL in laparoscopic gastrectomy combined with SNNS is superior in multiple categories compared to conventional laparoscopic distal gastrectomy in patients with stage I gastric cancer.

\section{Compilance with Ethical Standards}

Conflicts of Interest The authors have no conflicts of interest to declare.

\section{References}

1. Kim J-P, Lee J-H, Kim S-J, Yu H-J, Yang H-K. Clinicopathologic characteristics and prognostic factors in 10783 patients with gastric cancer. Gastric Cancer. 1998;1(2):125-33. https:// doi.org/10.1007/s101200050006.

2. Guideline Committee of the Korean Gastric Cancer Association DWG Review. Korean Practice Guideline for Gastric Cancer 2018: an Evidence-based, Multi-disciplinary Approach. J 
Gastric Cancer. 2019;19(1):1-48. doi: https://doi.org/10.5230/ jgc.2019.19.e8.

3. Japanese gastric cancer treatment guidelines 2014 (ver. 4). Gastric Cancer. 2017;20(1):1-19. Epub 2016/06/28. doi: https://doi. org/10.1007/s10120-016-0622-4.

4. Kurokawa Y, Doki Y, Mizusawa J, Terashima M, Katai H, Yoshikawa $\mathrm{T}$, et al. Bursectomy versus omentectomy alone for resectable gastric cancer (JCOG1001): a phase 3, open-label, randomised controlled trial. Lancet Gastroenterol Hepatol. 2018;3(7):460-8. https://doi.org/10.1016/S2468-1253(18)30090-6.

5. Sasako M, Sano T, Yamamoto S, Kurokawa Y, Nashimoto A, Kurita A, et al. D2 lymphadenectomy alone or with paraaortic nodal dissection for gastric cancer. N Engl J Med. 2008;359(5):453-62. https://doi.org/10.1056/NEJMoa0707035.

6. Kim HH, Han SU, Kim MC, Kim W, Lee HJ, Ryu SW, et al. Effect of laparoscopic distal gastrectomy vs open distal gastrectomy on long-term survival among patients with stage I gastric cancer: The KLASS-01 Randomized Clinical Trial. JAMA Oncol. 2019;5(4):506-13. https://doi.org/10.1001/jamaoncol.2018.6727.

7. Morton DL, Wen DR, Wong JH, Economou JS, Cagle LA, Storm FK, et al. Technical details of intraoperative lymphatic mapping for early stage melanoma. Arch Surg. 1992;127(4):392-9. https ://doi.org/10.1001/archsurg.1992.01420040034005.

8. Kett K, Varga G, Lukács L. Direct lymphography of the breast. Lymphology. 1970;3(1):2-12.

9. Krag DN, Weaver DL, Alex JC, Fairbank JT. Surgical resection and radiolocalization of the sentinel lymph node in breast cancer using a gamma probe. Surg Oncol. 1993;2(6):335-9. https://doi. org/10.1016/0960-7404(93)90064-6.

10. Kitagawa Y, Fujii H, Mukai M, Kubota T, Ando N, Watanabe $\mathrm{M}$, et al. The role of the sentinel lymph node in gastrointestinal cancer. Surg Clin North Am. 2000;80(6):1799-809. https://doi. org/10.1016/s0039-6109(05)70262-0.

11. Kim DW, Jeong B, Shin IH, Kang U, Lee Y, Park YS, et al. Sentinel node navigation surgery using near-infrared indocyanine green fluorescence in early gastric cancer. Surg Endosc. 2019;33(4):1235-43. https://doi.org/10.1007/s00464-018-6401-z.

12. Park DJ, Park YS, Son SY, Lee JH, Lee HS, Park YS, et al. Longterm oncologic outcomes of laparoscopic sentinel node navigation surgery in early gastric cancer: a single-center, single-arm, phase II trial. Ann Surg Oncol. 2018;25(8):2357-65. https://doi. org/10.1245/s10434-018-6523-5.

13. Shrive FM, Stuart H, Quan H, Ghali WA. Dealing with missing data in a multi-question depression scale: a comparison of imputation methods. BMC Med Res Methodol. 2006;6:57. https://doi. org/10.1186/1471-2288-6-57.

14. Nunobe S, Sasako M, Saka M, Fukagawa T, Katai H, Sano T. Symptom evaluation of long-term postoperative outcomes after pylorus-preserving gastrectomy for early gastric cancer. Gastric Cancer. 2007;10(3):167-72. https://doi.org/10.1007/s1012 0-007-0434-7.
15. Fujita J, Takahashi M, Urushihara T, Tanabe K, Kodera Y, Yumiba T, et al. Assessment of postoperative quality of life following pylorus-preserving gastrectomy and Billroth-I distal gastrectomy in gastric cancer patients: results of the nationwide postgastrectomy syndrome assessment study. Gastric Cancer. 2016;19(1):302-11. https://doi.org/10.1007/s10120-015-0460-9.

16. Yaguchi $Y$, Tsujimoto $H$, Hiraki S, Ito N, Nomura S, Horiguchi H, et al. Long-term outcome following sentinel node navigation surgery for cT1 gastric cancer. Mol Clin Oncol. 2019;10(6):615-8. https://doi.org/10.3892/mco.2019.1833.

17. Kitagawa Y, Takeuchi H, Takagi Y, Natsugoe S, Terashima M, Murakami N, et al. Sentinel node mapping for gastric cancer: a prospective multicenter trial in Japan. J Clin Oncol. 2013;31(29):3704-10. https://doi.org/10.1200/jco.2013.50.3789.

18. Isozaki H, Matsumoto S, Murakami S, Takama T, Sho T, Ishihara K, et al. Diminished gastric resection preserves better quality of life in patients with early gastric cancer. Acta Med Okayama. 2016;70(2):119-30. https://doi.org/10.18926/amo/54191.

19. Wu CW, Chiou JM, Ko FS, Lo SS, Chen JH, Lui WY, et al. Quality of life after curative gastrectomy for gastric cancer in a randomised controlled trial. Br J Cancer. 2008;98(1):54-9. https:// doi.org/10.1038/sj.bjc.6604097.

20. Kim YI, Kim YA, Kim CG, Ryu KW, Kim YW, Sim JA, et al. Serial intermediate-term quality of life comparison after endoscopic submucosal dissection versus surgery in early gastric cancer patients. Surg Endosc. 2018;32(4):2114-22. https://doi. org/10.1007/s00464-017-5909-y.

21. Okubo K, Arigami T, Matsushita D, Sasaki K, Kijima T, Noda M, et al. Evaluation of postoperative quality of life by PGSAS- 45 following local gastrectomy based on the sentinel lymph node concept in early gastric cancer. Gastric Cancer. 2020;23(4):746-53. https://doi.org/10.1007/s10120-020-01047-7.

22. Yaguchi Y, Tsujimoto H, Kumano I, Takahata R, Matsumoto $\mathrm{Y}$, Yoshida $\mathrm{K}$, et al. Sentinel node navigation surgery attenuates the functional disorders in early gastric cancer. Oncol Reports. 2012;27(3):643-9. https://doi.org/10.3892/or.2011.1568.

23. Park JY, Kim Y-W, Ryu KW, Nam B-H, Lee YJ, Jeong SH, et al. Assessment of laparoscopic stomach preserving surgery with sentinel basin dissection versus standard gastrectomy with lymphadenectomy in early gastric cancer-A multicenter randomized phase III clinical trial (SENORITA trial) protocol. BMC Cancer. 2016;16:340-. doi: https://doi.org/10.1186/s12885-016-2336-8. PubMed PMID: 27246120.

Publisher's Note Springer Nature remains neutral with regard to jurisdictional claims in published maps and institutional affiliations. 